\title{
Hypertree-Width and Related Hypergraph Invariants
}

\author{
Isolde Adler ${ }^{1}$, Georg Gottlob ${ }^{2 \dagger}$ and Martin Grohe ${ }^{3}$ \\ ${ }^{1}$ Abteilung für Mathematische Logik, Albert-Ludwigs-Universität Freiburg, Eckerstr. 1, 79104 Freiburg, Germany \\ ${ }^{2}$ Institut für Informationssysteme, TU Wien, Favoritenstr. 9-11/184-2, A-1040 Wien, Austria \\ ${ }^{3}$ Institut für Informatik, Humboldt-Universität zu Berlin, Unter den Linden 6, 10099 Berlin, Germany
}

\begin{abstract}
We study the notion of hypertree-width of hypergraphs. We prove that, up to a constant factor, hypertree-width is the same as a number of other hypergraph invariants that resemble graph invariants such as bramble-number, branchwidth, linkedness, and the minimum number of cops required to win Seymour and Thomas's robber and cops game.
\end{abstract}

Keywords: hypergraphs, tree decompositions, hypertree width

\section{Introduction}

Tree-width of graphs is a well studied notion, which plays an important role in structural graph theory and has many algorithmic applications. Various other graph invariants are known to be the same or within a constant factor of tree-width, for example, the bramble number or tangle number of a graph [4, 5], the branch-width [5], the linkedness [4], and the number of cops required to win the robber and cops game on the graph [6]. Several of these notions may be viewed as measures for the global connectivity of a graph. The various equivalent characterisations of tree-width show that it is a natural and robust notion.

Formally, let us call two graph or hypergraph invariants $I$ and $J$ equivalent if they are within a constant factor of each other, that is, if there are constants $c, d>0$ such that for all graphs or hypergraphs $G$ we have $c \cdot I(G) \leq J(G) \leq d \cdot I(G)$.

Tree decompositions and tree-width can be generalized to hypergraphs in a straightforward manner; the tree-width of a hypergraph is equal to the tree-width of its primal graph. Motivated by algorithmic problems from database theory and artificial intelligence, Gottlob, Leone, and Scarcello [2] introduced the hypertree-width of a hypergraph. Hypertree-width is based on the same tree decompositions as treewidth, but the width is measured differently. Essentially, the hypertree-width is the minimum number of hyperedges needed to cover all blocks of a tree decomposition. The blocks (or bags, parts) of a tree decomposition $\left(T,\left(B_{t}\right)_{t \in V(T)}\right)$ of a hypergraph $H=(V, E)$ are the sets $B_{t} \subseteq V$ for the tree-nodes $t$, and a block $B_{t}$ is covered by the hyperedges $e_{1}, \ldots, e_{k} \in E$ if $B_{t} \subseteq \bigcup_{i=1}^{k} e_{i}$. Hypertree-width is always

\footnotetext{
${ }^{\dagger}$ Georg Gottlob's work was supported by the Austrian Science Fund (FWF) under Project N. P17222-N04 'Complementary Approaches to Constraint Satisfaction'.
} 
bounded by one plus the tree-width [1]. ${ }^{\ddagger}$ Conversely, the hypergraph consisting of a single hyperedge with $n$ vertices has tree-width $n-1$ and hypertree-width 1 , thus tree-width and hypertree-width are not equivalent. It can also be shown that hypertree-width is not equivalent to the tree-width of the dual graph or the bipartite incidence graph of a hypergraph.

Unfortunately, we have not yet given the full definition of hypertree-width; the notion we have defined so far is called generalized hypertree-width. Hypertree-width is defined by adding a technical condition, the so-called special condition, that restricts the way blocks can be covered by hyperedges. A hypertree decomposition consists of a tree decomposition together with an appropriate cover of the blocks by edges. We will give the full technical definition in Section 5. The special condition is needed to prove that for fixed $k$, hypergraphs of hypertree-width $k$ can be recognized and hypertree decompositions of width $k$ can be computed in polynomial time. As an application, Gottlob, Leone, and Scarcello [2] showed that constraint satisfaction problems whose instances have an underlying hypergraph of bounded hypertree-width can be solved in polynomial time. Similarly, conjunctive database queries whose underlying hypergraph has bounded hypertree-width can be evaluated in polynomial time.

Despite the technical special condition, hypertree-width seems to be a quite natural notion. In [3], Gottlob, Leone, and Scarcello characterise hypertree-width in terms of a game that is defined like Seymour and Thomas's robber and cops game on graphs, except that instead of cops occupying vertices, the robber now has to escape marshals occupying hyperedges. Unfortunately, this characterisation also has a small technical defect: The marshals are required to play monotonically, that is, they have to shrink the escape space of the robber in each move. It can be shown that there are hypergraphs where this reduces the strength of the marshals, that is, $k$ marshals have a winning strategy, but no monotone winning strategy [1].

In this paper, we prove that the invariants hypertree-width, generalized hypertree-width, minimum number of marshals with a monotone winning strategy, and minimum number of marshals with a winning strategy are all equivalent. More precisely, they are all within a constant factor of $(3+\epsilon)$ from one another. Furthermore, we introduce invariants for measuring the global connectivity of a hypergraph that resemble bramble number, tangle number, branch-width, and linkedness of a graph and prove them all to be equivalent to hypertree-width. Our results show that hypertree-width is a similarly robust hypergraph invariant as tree-width is for graphs. Furthermore, they imply that the algorithmic applications of hypertree-width can be extended to generalized hypertree-width.

Due to space limitations, in this extended abstract we omit tangles and branch-decompositions of hypergraphs and the related notions of width. We also have to omit some of the proofs.

\section{Preliminaries}

A hypergraph is a pair $H=(V(H), E(H))$, consisting of a nonempty set $V(H)$ of vertices, and a set $E(H)$ of subsets of $V(H)$, the hyperedges of $H$. We only consider finite hypergraphs. Graphs are hypergraphs in which all hyperedges have two elements.

For a hypergraph $H$ and a set $X \subseteq V(H)$, the subhypergraph induced by $X$ is the hypergraph $H[X]=$ $(X,\{e \cap X \mid e \in E(H)\})$. We let $H \backslash X:=H[V(H) \backslash X]$. The primal graph of a hypergraph $H$ is the graph

$$
\underline{H}=(V(H),\{\{v, w\} \mid v \neq w, \text { there exists an } e \in E(H) \text { such that }\{v, w\} \subseteq e\}) .
$$

\footnotetext{
$\ddagger$ We do not require isolated vertices to be contained in any block of a tree decomposition, so we can disregard them.
} 
A hypergraph $H$ is connected if $\underline{H}$ is connected. A set $C \subseteq V(H)$ is connected (in $H$ ) if the induced subhypergraph $H[C]$ is connected, and a connected component of $H$ is a maximal connected subset of $V(H)$. A sequence of nodes of $V(H)$ is a path of $H$ if it is a path of $\underline{H}$.

A tree decomposition of a hypergraph $H$ is a tuple $(T, B)$, where $T$ is a tree and $B=\left(B_{t}\right)_{t \in V(T)}$ a family of subsets of $V(H)$ such that for each $e \in E(H)$ there is a node $t \in V(T)$ such that $e \subseteq B_{t}$, and for each $v \in V(H)$ the set $\left\{t \in V(T) \mid v \in B_{t}\right\}$ is connected in $T$.

We assume the tree $T$ in a tree decomposition to be rooted. For every node $t, T_{t}$ denotes the rooted subtree of $T$ with root $t$.

\section{From separators and hyperlinkedness ...}

Let $H$ be a hypergraph, $M \subseteq E(H)$ and $C \subseteq V(H)$. $C$ is $M$-big, if it intersects more than half of the edges of $M$, that is,

$$
|\{e \in M \mid e \cap C \neq \emptyset\}|>\frac{|M|}{2} .
$$

Note that if $S \subseteq E(H)$, then $H \backslash \bigcup S$ has at most one $M$-big connected component.

Let $k \geq 0$ be an integer. A set $M \subseteq E(H)$ is $k$-hyperlinked, if for any set $S \subseteq E(H)$ with $|S|<k$, $H \backslash \bigcup S$ has an $M$-big component. The largest $k$ for which $H$ contains a $k$-hyperlinked set is called hyperlinkedness of $H$, hlink $(H)$. Hyperlinkedness is an adaptation of the linkedness of a graph [4] to our setting.

A set $S \subseteq E(H)$ is a balanced separator for a set $M \subseteq E(H)$ if $H \backslash \bigcup S$ has no $M$-big connected component. Observe that hlink $(H) \leq k$ if and only if every $M \subseteq E(H)$ has a balanced separator of size at most $k$.

Example 1. Let $H:=(\{1,2,3,4,5\},\{\{1,2\},\{1,3,5\},\{1,4\},\{2,5\},\{4,5\}\})$.

- Let $M_{1}:=E(H)$. Then $S:=\{\{1,3,5\}\}$ is a balanced separator of size 1 for $M_{1}$.

- $M_{2}:=\{\{1,2\}\{1,3,5\},\{2,5\}\}$ has no balanced separator of size 1 .

- $\operatorname{hlink}(H)=2$.

\section{$4 \quad \ldots$ to brambles ...}

Next, we adapt the bramble number of a graph [4] to our hypergraph context.

Let $H$ be a hypergraph. Sets $X_{1}, X_{2} \subseteq V(H)$ touch if $X_{1} \cap X_{2} \neq \emptyset$ or or there exists an $e \in E(H)$ such that $e \cap X_{1} \neq \emptyset$ and $e \cap X_{2} \neq \emptyset$. A bramble of $H$ is a set $B$ of pairwise touching connected subsets of $V(H)$. The hyper-order of a bramble $B$ is the least integer $k$ such that there exists a set $R \subseteq E(H)$ with $|R|=k$ and $\bigcup R \cap X \neq \emptyset$ for all $X \in B$. The hyperbramble number hbramble-no $(H)$ of $H$ is the maximum of the hyper-orders of all brambles of $H$.

The following lemma follows from the observation that a separator for a bramble has to intersect all elements of the bramble.

Lemma 2. hlink $(H) \leq$ hbramble-no $(H)$ for every hypergraph $H$.

We cannot obtain equality here, as the following example shows:

Example 3. hlink $\left(K_{5}\right) \leq 2<3 \leq$ hbramble-no $\left(K_{5}\right)$, where $K_{5}$ denotes the 5-clique. 


\section{$5 \quad \ldots$ and via marshals and hypertree-width ...}

Let $H$ be a hypergraph and $k \geq 0$ an integer. The Robber and $k$ Marshals Game on $H$ is played by two players, I and II, on the hypergraph $H$. Player I plays $k$ marshals and player II plays the robber. The marshals move on the hyperedges of $H$, trying to catch the robber. In each move, some of the marshals fly in helicopters to new hyperedges. The robber moves on the vertices of $H$. He sees where the marshals will be landing and quickly tries to escape running arbitrarily fast along paths of $H$, not being allowed to run through a vertex that is occupied by a marshal before and after the flight. Player I's objective is to land a marshal via helicopter on a hyperedge containing the vertex occupied by the robber. Player II tries to elude capture. In the monotone version of the game, the marshals have to make sure, that in each step the robber's escape space decreases. The (monotone) marshal-width of a hypergraph $H, \operatorname{mw}(H)$ (and mon-mw $(H)$, respectively), is the least number $k$ of marshals that have a (monotone) winning strategy in the robber and $k$ marshals game played on $H$ (see [1], [3] for more precise definitions). For instance, the hypergraph $H$ from Example 1 satisfies $\operatorname{mw}(H)=\operatorname{mon}-\operatorname{mw}(H)=2$.

Lemma 4. hbramble-no $(H) \leq \operatorname{mw}(H)$ for every hypergraph $H$.

Proof: Let $B$ be a bramble in $H$ of hyper-order $k=$ hbramble-no $(H)$. We show that the robber can avoid being caught by $k-1$ marshals: Suppose the game is in position $(M, C)$, where $M \subseteq E(H)$ with $|M| \leq k-1$ and $C$ the component of $H \backslash(\bigcup M)$ containing a set $B_{M} \in B$ such that $B_{M} \cap(\bigcup M)=\emptyset(M$ is the marshal's position and $C$ is the robber's position). Note that $C$ is unique, since any two elements of $B$ touch. Now the marshals move to $N \subseteq E(H)$. Then the robber moves to the component $D$ of $H \backslash(\bigcup N)$ containing a $B_{N} \in B$ with $B_{N} \cap(\bigcup N)=\emptyset$. This is possible, since $B_{M}$ and $B_{N}$ touch in $H \backslash(\bigcup M \cup \bigcup N)$. Thus, the robber can escape.

Let $H$ be a hypergraph. A generalized hypertree decomposition of $H$ is a triple $(T, B, C)$, where $(T, B)$ is a tree decomposition of $H$ and $C=\left(C_{t}\right)_{t \in V(T)}$ is a family of subsets of $E(H)$ such that for every $t \in V(T)$ we have $B_{t} \subseteq \bigcup C_{t}$. The width of $(T, B, C)$ is $\min \left\{\left|C_{t}\right| \mid t \in V(T)\right\}$. The generalized hypertree-width $\operatorname{ghw}(H)$ of $H$ is the minimum of the widths of the hypertree decompositions of $H$.

A hypertree decomposition of $H$ is a generalized hypertree decomposition $(T, B, C)$ that satisfies the following special condition: $\left(\bigcup C_{t}\right) \cap \bigcup_{u \in V\left(T_{t}\right)} B_{u} \subseteq B_{t}$ for all $t \in V(T)$. Recall that $T_{t}$ denotes the subtree of the $T$ with root $t$. The hypertree-width $\mathrm{hw}(H)$ of $H$ is the minimum of the widths of all hypertree decompositions of $H$.

Theorem $5([1,3]) \cdot \operatorname{mw}(H) \leq \operatorname{ghw}(H) \leq \mathrm{hw}(H)=$ mon-mw $(H)$ for every hypergraph $H$.

\section{6 ... back to separators}

Let $H$ be a hypergraph and $X \subseteq V(H)$. For every connected component $C$ of $H \backslash X$ we let

$$
\partial C=\{v \in X \mid \text { there is a hyperedge } e \in E(H) \text { with } v \in e \text { and } e \cap C \neq \emptyset\} .
$$

Lemma 6. Let $k \geq 1$, $H$ a hypergraph with $\operatorname{hlink}(H) \leq k$, and $M \subseteq E(H)$ with $|M| \leq 2 k+1$.

Then there exists a set $X^{\prime} \subseteq E(H)$ with $M \subseteq X^{\prime}$ and $\left|X^{\prime}\right| \leq 3 k+1$ such that for all components $R^{\prime}$ of $H \backslash\left(\bigcup X^{\prime}\right)$ there exists a subset $M^{\prime} \subseteq X^{\prime}$ with $\left|M^{\prime}\right| \leq 2 k$ and $\partial R^{\prime} \subseteq \bigcup M^{\prime}$. 
Proof: Let $S$ be a balanced separator for $M$ with $|S| \leq k$ and $X^{\prime}:=M \cup S$. Then $M \subseteq X^{\prime}$ and $\left|X^{\prime}\right| \leq 3 k+1$. Let $R^{\prime}$ be a component of $H \backslash\left(\bigcup X^{\prime}\right)$. Then $R^{\prime} \subseteq C$ for a component $C$ of $H \backslash(\bigcup S)$. Since $S$ is a balanced separator for $M, C$ is not $M$-big, i. e.

$$
|\{e \in M \mid e \cap C \neq \emptyset\}| \leq \frac{|M|}{2}=\frac{2 k+1}{2} .
$$

Let $M^{\prime}:=S \cup\{e \in M \mid e \cap C \neq \emptyset\}$, then $M^{\prime} \subseteq X^{\prime}$ and $\left|M^{\prime}\right| \leq k+k$. Furthermore,

$$
\partial R^{\prime} \subseteq \bigcup\left\{e \in X^{\prime} \mid e \cap C \neq \emptyset\right\} \subseteq \bigcup S \cup \bigcup\{e \in M \mid e \cap C \neq \emptyset\}=\bigcup M^{\prime} .
$$

Lemma 7. mon-mw $(H) \leq 3 \cdot \operatorname{hlink}(H)+1$ for every hypergraph $H$.

Proof: Let hlink $(H)=k$. The marshals can make sure that each position $(X, R)$ satisfies $|X| \leq 3 k+1$ and $\partial R \subseteq \bigcup M$ for an $M \subseteq X$ with $|M| \leq 2 k$ :

Suppose this is true for $(X, R)$. Choose $e \in E(H)$ with $e \cap R \neq \emptyset$. Application of Lemma 6 to $M \cup\{e\}$ yields $X^{\prime}$, s. t. for each possible escape space $R^{\prime}$ with respect to $X^{\prime}$ there exists an $M^{\prime} \subseteq X^{\prime}$ with $\left|M^{\prime}\right| \leq 2 k$ and $\partial R^{\prime} \subseteq \bigcup M^{\prime}$.

\section{Putting things together}

Theorem 8. Let $H$ be a hypergraph. Then

$$
\operatorname{hlink}(H) \leq \operatorname{hbramble-no}(H) \leq \operatorname{mw}(H) \leq \operatorname{ghw}(H) \leq \operatorname{hw}(H) \leq 3 \cdot \operatorname{hlink}(H)+1 .
$$

Example 3 shows that there is a hypergraph $H$ for which the first inequality is strict. It is proved in [1] that there are hypergraphs for which the third and fourth inequality, respectively, are strict. The fifth inequality is strict for the trivial hypergraph consisting of one vertex and one hyperedge containing it. We do not know if there is a hypergraph for which the second inequality is strict.

In the full paper, we also prove that hbramble-no $(H) \leq 2 \cdot \operatorname{hlink}(H)$. Furthermore, we introduce the hypertangle number htangle-no $(H)$ and the hyperbranch-width of a hypergraph $H$ and prove that

$$
\text { htangle-no }(H) \leq \text { hbramble-no }(H) \leq 3 \cdot \text { htangle-no }(H)
$$

and hbranch-width $(H) \leq \operatorname{ghw}(H) \leq 2 \cdot \operatorname{hbranch}-\operatorname{width}(H)$.

\section{References}

[1] I. Adler. Marshals, monotone marshals, and hypertree-width. Journal of Graph Theory, 47:275-296, 2004.

[2] G. Gottlob, N. Leone, and F. Scarcello. Hypertree decompositions and tractable queries. Journal of Computer and System Sciences, 209:1-45, 2002.

[3] G. Gottlob, N. Leone, and F. Scarcello. Robbers, marshals, and guards: Game theoretic and logical characterizations of hypertree width. Journal of Computer and System Sciences, 66:775-808, 2003. 
[4] B. Reed. Tree width and tangles: A new connectivity measure and some applications. In R.A. Bailey, editor, Surveys in Combinatorics, volume 241 of LMS Lecture Note Series, pages 87-162. Cambridge University Press, 1997.

[5] N. Robertson and P.D. Seymour. Graph minors X. Obstructions to tree-decomposition. Journal of Combinatorial Theory, Series B, 52:153-190, 1991.

[6] P.D. Seymour and R. Thomas. Graph searching and a min-max theorem for tree-width. Journal of Combinatorial Theory, Series B, 58:22-33, 1993. 